\section{Reemplazo valvular mitral en edad pediátrica}

Diliz-Nava HS ${ }^{1}$, Pérez-Juárez $F^{2}$, Araujo-Martínez $A^{3}$, García-Benítez L ${ }^{4}$, Tamariz-Cruz $\mathrm{O}^{5}$, Palacios-Macedo-Quenot $\mathrm{A}^{6}$

\section{Resumen}

ANTECEDENTES: el reemplazo valvular mitral en pediatría es un procedimiento raro asociado con dificultades técnicas y clínicas únicas. Estudios recientes reportan mejores resultados, a corto y largo plazo, posteriores al procedimiento.

OBJETIVO: analizar la experiencia del reemplazo valvular mitral en el Instituto Nacional de Pediatría.

MATERIALES Y MÉTODOS: se revisaron los expedientes de los pacientes con reemplazo valvular mitral, en el Instituto Nacional de Pediatría, entre agosto del 2002 y agosto del 2012. Las variables de evaluación primaria fueron mortalidad, complicaciones de la anticoagulación y resultados a largo plazo. Se incluyó a doce pacientes, con mediana de edad de 12.5 años (tres pacientes menores de 5 años).

RESULTADOS: en 11 casos la anomalía mitral fue considerada congénita. La manifestación clínica más frecuente fue insuficiencia mitral. La mediana de la fracción de eyección del ventrículo izquierdo fue de $62 \%$ antes de la cirugía. Se colocó prótesis mecánica en 11 casos. Dos pacientes fallecieron en el postoperatorio inmediato, con supervivencia a 30 días de $83 \%$, sin reporte de ninguna muerte en el periodo de seguimiento. Un paciente presentó sangrado de tubo digestivo leve y dos arritmia auricular. No se reportaron eventos tromboembólicos ni necesidad de nueva intervención. La mediana del tiempo de seguimiento fue de 16.6 meses.

CONCLUSIÓN: en nuestras condiciones el reemplazo valvular mitral parece ser una buena opción para los pacientes que no pueden beneficiarse de la reparación, con resultados aceptables a corto y mediano plazos.

PALABRAS CLAVE: válvula mitral, enfermedad congénita de la válvula mitral, implantación de prótesis valvular cardiaca.

Acta Pediatr Mex. 2017 Jan;38(1):10-16.

\section{Mitral valve replacement in infants and children.}

Diliz-Nava HS ${ }^{1}$, Pérez-Juárez $F^{2}$, Araujo-Martínez $A^{3}$, García-Benítez L ${ }^{4}$, Tamariz-Cruz $\mathrm{O}^{5}$, Palacios-Macedo-Quenot $\mathrm{A}^{6}$

\section{Abstract}

BACKGROUND: Mitral valve replacement in children is a rare procedure associated to unique clinical and technical difficulties.
${ }^{1}$ Residente de Cirugía Cardiotorácica Pediátrica. ${ }^{2} J$ efe de Laboratorio de Ecocardiografía. ${ }^{3}$ Intensivista Cardiovascular. Adscrito a la Unidad de Cuidados Intensivos Cardiovasculares.

${ }^{4}$ Metodólogo, Manejo de la base de datos, División de Cirugía Cardiovascular.

${ }^{5}$ Jefe de Anestesiología Cardiovascular de Congénitos, División de Cirugía Cardiovascular.

${ }^{6} J$ efe de la División de Cirugía Cardiovascular.

Instituto Nacional de Pediatría, Ciudad de México.

Recibido: 15 de julio del 2015

Aceptado: 24 de mayo del 2016

Correspondencia

Dr. Alexis Palacios-Macedo-Quenot

apalaciosmacedo@icloud.com

Este artículo debe citarse como

Diliz-Nava HS, Pérez-Juárez F, Araujo-Martínez A, García-Benítez L, Tamariz-Cruz O, Palacios-MacedoQuenot A. Reemplazo valvular mitral en edad pediátrica. Acta Pediatr Mex. 2017;38(1):10-16. 
Recent studies suggest a better outcome on short and long term after MVR.

OBJECTIVE: The aim of this study is to analyze the experience with mitral valve replacement at the National Institute of Pediatrics (NIP) in Mexico City.

METHODS: On a retrospective basis, we reviewed the charts of pediatric patients who underwent MVR at the NIP from August 2002 to August 2012. Primary endpoints measured were mortality, anticoagulation complications and long-term outcomes.

RESULTS: Twelve patients underwent mitral valve replacement. Median age of 11.5 years, three patients were under 5 years. Mitral dysfunction was considered congenital in 11 and rheumatic in 1. The hemodynamic manifestation was mitral insufficiency in 8 cases, combined mitral stenosis and mitral insufficiency in 3, and pure stenosis in one. Mean left ventricular ejection fraction was $63 \%$ prior to surgery. Mechanical prosthesis was placed in 11 cases. One patient received a biological prosthesis. Two patients died in the immediate postoperative period, with a 30-day survival of $83 \%$. No mortality was reported in the follow up period. One patient had an episode of mild gastrointestinal bleeding and two patients had atrial arrhythmia. No thromboembolic events. There were no re-interventions. Median follow up time was 3 years.

CONCLUSION: In our conditions and population, mitral valve replacement seems a good option for patients who cannot benefit from repair, with acceptable results on a short and long term follow up.

KEYWORDS: mitral valve; congenital mitral valve disease; heart valve prosthesis implantation

\begin{abstract}
${ }^{1}$ Residente de Cirugía Cardiotorácica Pediátrica.

${ }^{2}$ Jefe de Laboratorio de Ecocardiografía.

${ }^{3}$ Intensivista Cardiovascular. Adscrito a la Unidad de Cuidados Intensivos Cardiovasculares.

${ }^{4}$ Metodólogo, Manejo de la base de datos, División de Cirugía Cardiovascular.

5 Jefe de Anestesiología Cardiovascular de Congénitos, División de Cirugía Cardiovascular.

${ }^{6}$ Jefe de la División de Cirugía Cardiovas-
\end{abstract} cular.

Instituto Nacional de Pediatría, Ciudad de México.

\section{Correspondence}

Dr. Alexis Palacios-Macedo-Quenot

apalaciosmacedo@icloud.com

\section{ANTECEDENTES}

Preservar el tejido nativo en cualquier tipo de cirugía valvular cardiaca permite su crecimiento $y$, potencialmente, mejor pronóstico a largo plazo. ${ }^{1}$ Cuando la reconstrucción falla o no es factible el reemplazo valvular es inevitable. ${ }^{2} \mathrm{EI}$ reemplazo valvular mitral es un procedimiento raro en niños, al que se asocian dificultades clínicas y técnicas específicas. ${ }^{3,4}$ Actualmente, dicha cirugía representa un procedimiento de bajo riesgo, incluso en pacientes pequeños, me- nores de dos años de edad, con baja mortalidad operatoria. ${ }^{2,4-6}$ Algunos de los factores reportados previamente con mayor impacto en morbilidad y mortalidad en pacientes sometidos a reemplazo valvular mitral son el flujo restrictivo que causará una prótesis pequeña durante el proceso de crecimiento, la necesidad de cambio valvular a largo plazo y de anticoagulación, en el caso de implantar una prótesis mecánica. ${ }^{7}$ Existen muy pocos reportes en la literatura sobre la experiencia en este procedimiento en pediatría en países en desarrollo. 


\section{OBJETIVO}

Analizar la experiencia del reemplazo valvular mitral en el Instituto Nacional de Pediatría.

\section{MATERIALES Y MÉTODOS}

Se trata de un estudio retrospectivo donde se revisaron los expedientes de todos los pacientes menores de 18 años, a quienes se les practicó reemplazo valvular mitral en el Instituto Nacional de Pediatría durante el periodo de agosto de 2002 a agosto de 2012. Los datos se obtuvieron de los expedientes clínicos, complementados con la información de la base de datos de la División de Cirugía Cardiovascular. Los resultados se expresaron utilizando proporciones y como medida de tendencia central medianas, máximos y mínimos. Para realizar el análisis estadístico se utilizó el programa STATA 12.2 para Windows ${ }^{\circledR}$. La supervivencia y las complicaciones secundarias a la anticoagulación se describieron usando curvas de Kaplan-Meier. Se considero un intervalo de confianza de $95 \%$ para la significancia estadística. No se pudo llevar a cabo un análisis multivariado debido al pequeño número de pacientes y variables.

\section{RESULTADOS}

\section{Datos preoperatorios}

Pacientes: 12 pacientes requirieron reemplazo valvular mitral, 6 mujeres y 6 hombres, con una mediana de edad de 12.5 años (mínimo 1.4 años y máximo de 18.5 años). Tres pacientes (25\%) tenían menos de 5 años de edad, un paciente $(8.3 \%)$ menos de 2 años. La mediana de edad al momento de la intervención fue de $32.2 \mathrm{~kg}$ (mínimo de 5.4 kg y máximo de 52 kg).

Ecocardiograma: diez pacientes (83\%) presentaron función ventricular izquierda normal antes de la cirugía. La mediana de la fracción de eyección fue de $62 \%$. Dos pacientes tuvieron fracción de eyección menor de 55\%.

Etiología e indicación para reemplazo valvular: la etiología de la enfermedad mitral fue congénita en 11 pacientes $(91 \%)$ y reumática en uno $(9 \%)$. La manifestación hemodinámica más frecuente fue insuficiencia mitral en 8 pacientes $(66 \%)$, estenosis/insuficiencia mitral en 3 (25\%) y estenosis mitral en 1 paciente $(8 \%)$.

Cirugías previas: cinco pacientes (41\%) tuvieron una cirugía cardiaca previa. Cierre de comunicación interventricular en dos, reemplazo valvular mitral en uno, plastía de la válvula mitral en uno y reparación de arco aórtico por coartación en otro.

Información quirúrgica

Cirugía y procedimientos concomitantes: la técnica quirúrgica incluyó esternotomía media, apoyo con circulación extracorpórea, canulación aórtica y bicaval, hipotermia moderada y aplicación repetida de cardioplegia anterógrada. Se utilizó abordaje transeptal en todos los pacientes para exponer la válvula mitral. Se escogió este abordaje debido al tamaño pequeño del corazón, aunado a la poca dilatación de la aurícula izquierda. La mediana del tiempo de circulación extracorpórea fue de 124.5 minutos (mínimo de 98 minutos y máximo de 400 minutos), así como la de pinzamiento aórtico fue de 98.5 minutos (mínimo de 98 minutos y máximo de 275 minutos). A once pacientes (91\%) se les colocó una prótesis mecánica St. Jude Medical ${ }^{\circledR}$ y a un paciente se le colocó una válvula biológica Carpentier-Edwards, Perimount ${ }^{\mathbb{B}}$. El rango de tamaño de las prótesis colocadas fue de 21-31 mm. Seis pacientes tuvieron otro tipo de defecto cardiaco, de los cuales sólo 4 requirieron de un procedimiento concomitante, incluida una reparación de doble salida de ventrículo derecho, plastía de la válvula tricúspide 
en dos pacientes y reparación de arco aórtico por coartación en uno.

\section{Datos postoperatorios}

Anticoagulación: en el postoperatorio inmediato se utilizó infusión de heparina hasta iniciar la vía oral. Nueve pacientes recibieron warfarina con el objetivo de mantener un INR de 2.5-3.5. Se utilizó aspirina en la paciente que recibió la válvula biológica. Dos pacientes sólo recibieron heparina debido al estado crítico, previo a su faIlecimiento en la Unidad de Cuidados Intensivos Cardiovasculares.

Seguimiento: se llevaron a cabo revisiones periódicas por un cardiólogo pediatra cada 3 a 6 meses. La mediana de seguimiento fue de 16.6 meses (mínimo de 5 días y máximo de 120.9 meses).

\section{Seguimiento}

Mortalidad operatoria: hubo dos muertes operatorias (18\%) dentro de los primeros 30 días posteriores al reemplazo valvular mitral.

Paciente 1: paciente masculino de 16 años, con diagnóstico de insuficiencia mitral y coartación de aorta. Mediante esternotomía media realizamos inicialmente la corrección de la coartación con anastomosis término terminal a $18^{\circ}$ con perfusión cerebral no selectiva. Tuvimos problemas de sangrado debido a múltiples colaterales que nacían de la aorta. Posteriormente, a $28^{\circ}$, intentamos la plastía mitral, debido a que el control ecocardiográfico mostraba aún insuficiencia mitral severa, se decidió colocar la prótesis mecánica. El paciente tuvo insuficiencia aórtica severa en el postoperatorio. De manera retrospectiva, el paciente no tuvo una adecuada valoración preoperatoria. El paciente falleció 16 días después de la cirugía por falla respiratoria. Este paciente tenía la fracción de eyección de ventrículo izquierdo más baja preoperatoria (44\%), así como el tiempo de circulación extracorpórea más prolongado de la serie (400 min).

Paciente 2: paciente masculino de 17 años de edad con diagnóstico de comunicación interventricular residual con insuficiencia tricuspídea y mitral. Tenía el antecedente de cierre de la comunicación interventricular y comisurotomía mitral a los 16 años de edad. Mediante reesternotomía media, a $28^{\circ}$, cerramos la comunicación interventricular residual, realizamos una anuloplastía de Kay en la válvula tricúspide y colocamos la prótesis mitral. Tuvo un tiempo de circulación extracorpórea de 123 minutos. Falleció al cuarto día postoperatorio debido a complicaciones sépticas.

Ningún paciente menor de 5 años falleció en el periodo de seguimiento. No hubo necesidad de nueva operación debido a complicaciones de la prótesis.

Complicaciones postoperatorias tempranas: las complicaciones tempranas se definieron como aquellas que ocurrieron en los primeros 30 días posteriores a la cirugía. Tres pacientes presentaron algún tipo de arritmia cardiaca; ninguno de ellos bloqueo. Dos presentaron fibrilación auricular con buena respuesta al tratamiento antiarrítmico. Uno falleció de fibrilación ventricular, probablemente como evento final de choque séptico. Otro desarrolló pancreatitis aguda, la cual se resolvió con tratamiento medico, sin complicaciones. Un último presentó derrame pericárdico en el postoperatorio, el cual se resolvió espontáneamente.

Eventos relacionados con la anticoagulación: no hubo eventos tromboembólicos en nuestra serie. Tres pacientes presentaron algún tipo de sangrado. Uno desarrolló epistaxis que requirió valoración por el Servicio de Otorrinolaringología; otro presentó equimosis y sólo requirió de 
ajuste de la dosis de warfarina, el tercer paciente desarrolló sangrado de tubo digestivo que remitió con la suspensión y ajuste de la warfarina (Figura 1).

Seguimiento a largo plazo: hasta el momento no ha habido ninguna muerte (Figura 2). En la última evaluación ecocardiográfica todos los pacientes tuvieron una adecuada función del ventrículo

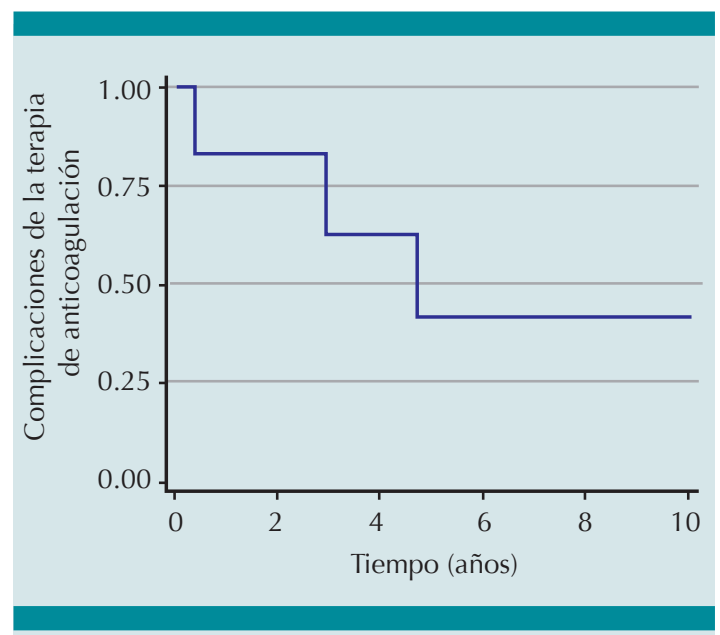

Figura 1. Tiempo libre de sangrado. Sin eventos tromboembólicos.

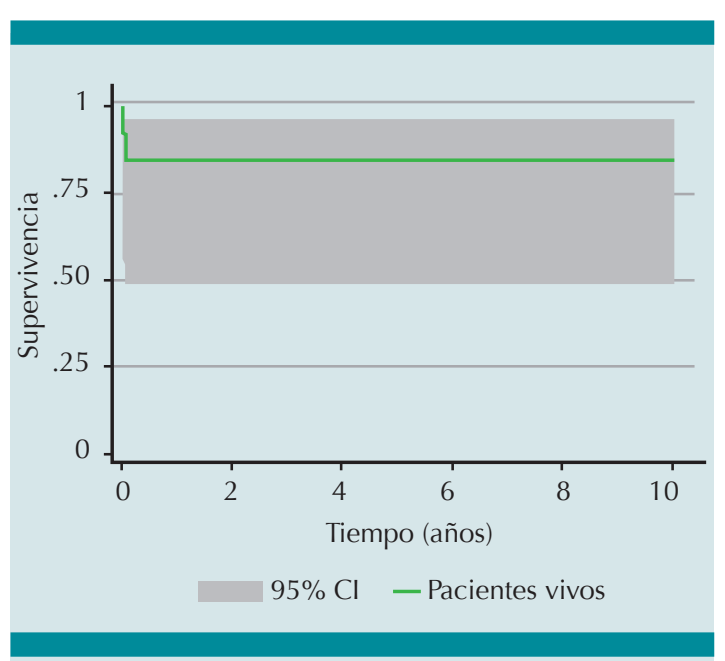

Figura 2. Curva Kaplan-Meier de supervivencia después del reemplazo valvular mitral. izquierdo y de la prótesis mitral. En el Cuadro 1 se resumen los resultados y seguimiento de todos los pacientes.

\section{DISCUSIÓN}

La cirugía reconstructiva ofrece los mejores resultados en los pacientes con enfermedad mitral congénita. En nuestra institución, como en la mayoría de los reportes en la literatura, la cirugía reconstructiva es el objetivo primario. En los casos donde la válvula mitral es muy displásica o la reparación ha fallado, el reemplazo valvular mitral es la única opción. ${ }^{3,4,8,9}$ Durante la exploración quirúrgica de las válvulas mitrales encontramos que $50 \%$ de las válvulas mitrales estaban de moderada a severamente displásicas, sin considerar la reparación como opción. Se utilizó una válvula mecánica St. Jude en la mayoría de los pacientes. Las ventajas de este tipo de válvula son: tener un buen perfil de flujo hemodinámico, menor potencial de obstrucción al tracto de salida del ventrículo izquierdo y mayor durabilidad. , $8,10-12^{2}$

Como lo sugieren el Dr. Alsoufi y su grupo, los homoinjertos y válvulas biológicas, aunque no son la primera opción, son un recurso válido en pacientes de sexo femenino y en pacientes con pobre apego al tratamiento con anticoagulantes. Otro grupo de pacientes candidatos para colocación de una válvula biológica son los que no tienen acceso a una monitorización adecuada de la anticoagulación. , $9,12,13$ En nuestra serie colocamos una válvula biológica en una paciente de 18 años con retraso moderado en el neurodesarrollo, de escasos recursos y con dificultad para llevar un adecuado control de anticoagulación.

Las complicaciones por sangrado fueron raras en nuestra serie, con sólo eventos menores. Esto puede deberse a una predominante mayor edad 


\begin{tabular}{|c|c|c|c|c|c|c|c|c|c|c|c|c|c|c|}
\hline$\#$ & Sexo & $\begin{array}{c}\text { Edad } \\
\text { (años) }\end{array}$ & $\begin{array}{l}\text { Peso } \\
\text { (kg) }\end{array}$ & Etiología & $\begin{array}{l}\text { Lesión } \\
\text { mitral }\end{array}$ & $\begin{array}{c}\text { Patología } \\
\text { asociada }\end{array}$ & $\begin{array}{c}\text { FEVI } \\
\text { (previa) }\end{array}$ & $\begin{array}{l}\text { CEC } \\
(\mathrm{min})\end{array}$ & $\begin{array}{c}\text { PinzAo } \\
\text { (mín) }\end{array}$ & $\begin{array}{c}\text { Prótesis } \\
\text { (tipo) }\end{array}$ & $\begin{array}{l}\text { Prótesis } \\
\text { (diáme- } \\
\text { tro } \mathrm{mm} \text { ) }\end{array}$ & $\begin{array}{c}\text { Procedi- } \\
\text { miento } \\
\text { conco- } \\
\text { mitante }\end{array}$ & $\begin{array}{c}\text { Segui- } \\
\text { miento } \\
\text { (meses) }\end{array}$ & $\begin{array}{l}\text { Compli- } \\
\text { cación }\end{array}$ \\
\hline 1 & $\mathrm{~F}$ & 17.9 & 40 & Congénita & EM & IA & 48 & 152 & 85 & Mecánica & 27 & No & 7.2 & FA \\
\hline 2 & M & 15.7 & 52 & Congénita & $I M$ & No & 58.4 & 126 & 102 & Mecánica & 29 & No & 27.5 & $\begin{array}{l}\text { Pancrea- } \\
\text { titis }\end{array}$ \\
\hline 3 & $\mathrm{~F}$ & 7.6 & 16 & Congénita & $I M$ & DSVD & 87 & 201 & 171 & Mecánica & 23 & $\begin{array}{c}\text { Correc- } \\
\text { ción } \\
\text { DSVD }\end{array}$ & 56.5 & $\begin{array}{c}\text { Sangrado/ } \\
\text { DP }\end{array}$ \\
\hline 4 & $\mathrm{~F}$ & 3.9 & 12.3 & Congénita & IM & No & 63 & 100 & 68 & Mecánica & 23 & No & 35.3 & $\begin{array}{c}\text { Sangrado/ } \\
\text { FA }\end{array}$ \\
\hline 5 & M & 1.4 & 5.4 & Congénita & IM & No & 68 & 123 & 114 & Mecánica & 23 & No & 4.4 & Sangrado \\
\hline 6 & $\mathrm{~F}$ & 18.5 & 32.5 & Congénita & IM & No & 71 & 135 & 105 & Biológica & 25 & No & 11.1 & No \\
\hline 7 & M & 9.4 & 32 & Congénita & IM & No & 64 & 98 & 83 & Mecánica & 29 & No & 21.2 & No \\
\hline 8 & M & 2.3 & 7.3 & Congénita & EM/IM & No & 73 & 106 & 75 & Mecánica & 21 & No & 67.7 & No \\
\hline 9 & $\mathrm{~F}$ & 9.2 & 20 & $\begin{array}{l}\text { Reumá- } \\
\text { tica }\end{array}$ & $\mathrm{EM} / \mathrm{IM}$ & IT & 57 & 120 & 95 & Mecánica & 21 & $\begin{array}{l}\text { Anulo- } \\
\text { plastía } \\
\text { Kay }\end{array}$ & 120.9 & No \\
\hline 10 & M & 16.2 & 40 & Congénita & IM & $\begin{array}{c}\text { Coartación } \\
\text { aorta }\end{array}$ & 44 & 400 & 275 & Mecánica & 29 & $\begin{array}{c}\text { Repara- } \\
\text { ción arco } \\
\text { aórtico }\end{array}$ & 0.5 & Muerte \\
\hline 11 & M & 17.9 & 50 & Congénita & $I M$ & CIV & 60 & 123 & 90 & Mecánica & 31 & $\begin{array}{c}\text { Anulo- } \\
\text { plastía } \\
\text { Kay + } \\
\text { cierre } \\
\text { CIV }\end{array}$ & 0.1 & $\begin{array}{c}\text { FV/Muer- } \\
\text { te }\end{array}$ \\
\hline 12 & $F$ & 17.6 & 47 & Congénita & $E M / I M$ & No & 61 & 146 & 102 & Mecánica & 29 & No & 12.1 & FA \\
\hline
\end{tabular}

CEC: circulación extracorpórea; CIV: comunicación interventricular; DP: derrame pericárdico; DSVD: doble salida de ventrículo derecho; EM: estenosis mitral; FA: fibrilación auricular; FEVI: fracción de eyección de ventrículo izquierdo; IA: insuficiencia aórtica; IM: insuficiencia mitral; FV: fibrilación ventricular; PinzAo: pinzamiento aórtico.

que correlaciona con un mayor apego y menor variación en las dosis del anticoagulante. Es de notar que ningún paciente menor de 5 años presentó eventos de sangrado.

La mortalidad temprana posterior a reemplazo valvular mitral se encuentra entre 11 y $36 \%$. La mortalidad reportada, cuando la cirugía se realiza en los dos primeros años de vida, se incrementa de 26 hasta $52 \% .^{13,14}$ Se han reportado múltiples factores asociados a mortalidad en pacientes operados de reemplazo valvular mitral. Henaine y sus colaboradores encontraron que el diagnóstico de canal auriculoventricular completo, el síndrome de Shone, una relación tamaño de prótesis/peso mayor y la posición supra anular de la prótesis son factores asociados con mortalidad temprana. ${ }^{2}$ La morfología de la válvula mitral, un mayor tiempo de circulación extracorpórea, síndromes extracardiacos, edad menor de 2 años, también son factores reportados por otros autores. ${ }^{3,5,6,15}$ 
En nuestro estudio sólo dos pacientes tuvieron muerte temprana (18\%), ambos adolescentes. Un paciente tuvo el tiempo de circulación extracorpórea más largo de la serie, mientras que el otro paciente falleció de complicaciones sépticas. El reemplazo de la válvula mitral en los primeros años de vida es un gran reto. El anillo mitral pequeño, la función cardiaca disminuida en el postoperatorio y la dificultad del ajuste de la terapia anticoagulante representan varios problemas. ${ }^{14,16}$ Actualmente, la supervivencia en pacientes menores de 5 años está mejorando. ${ }^{17}$ Tres pacientes en nuestra serie tenían menos de 5 años, uno de ellos menos de 2 años. Todos ellos se reportan con buen resultado funcional, vivos y libres de complicaciones mayores.

Este estudio expone la experiencia de un centro pediátrico en reemplazo de la válvula mitral. Es un análisis retrospectivo, con un número pequeño de pacientes, con un período de seguimiento no lo suficientemente largo para ser concluyente; además de estar sujeto a las deficiencias asociadas a un estudio retrospectivo. Sin embargo, el reemplazo de la válvula mitral parece ser una buena opción para pacientes que no pudieron beneficiarse de una plastía valvular en nuestro instituto, con resultados similares a otras series con una calidad de vida aceptable.

\section{REFERENCIAS}

1. Tiete AR, Sachweh JS, Groetzner J, Gulbins H, Muehler EG, Messmer BJ, et al. Systemic mechanical heart valve replacement in children under 16 years of age. Clin Res Cardiol. 2006;95:281-8.

2. Henaine $R$, Roubertie $F$, Vergnat $M$, Ninet J. Valve replacement in children: A challenge for a whole life. Arch Cardiovasc Dis. 2012;105:517-28.

3. Kojori F, Chen R, Calderone C, Merklinger SL, Azakie A, Williams WG, et al. Outcomes of mitral valve replacement in children: A competing-risk analysis. J Thorac Cardiovasc Surg. 2004;128:703-9.

4. Agarwal S, Mukherjee K, Geelani M, Satsangi DK, Pratap $\mathrm{H}$, Banarjee A. Mitral valve replacement in the pediatric age group- A Single Institution Experience. Ind J Thorac Cardiovasc Surg. 2009;25:7-11.

5. Erez E, KanterKR, Isom E, Williams W, Tam V. Mitral valve replacement in children. J Heart Valve Dis. 2003;12:25-30.

6. Rafii DY, Davies RR, Carroll SJ, Quaegebeur JM, Chen JM. Age less than two years is not a risk factor for mortality after mitral valve replacement in children. Ann Thorac Surg. 2011;91:1228-34.

7. Becker P, Frangini P, Arretz C, Heusser F, Urcelay G, Zalaquett $R$, et al. Reemplazo valvular mitral y aórtico en niños: Resultados durante la última década con prótesis de última generación. Rev Chil Pediatr, 2005;76:375-83.

8. Günther T, Mazzitelli D, Schreiber Ch, Wottke M, Paek SU, Meissner $\mathrm{H}$, et al. Mitral valve replacement in children under 6 years of age. Eur J Cardiothorac Surg, 2000;17:426-30.

9. Alexiou Ch, Galogavrou M, Chen Q, McDonald A, Salmon A, Keeton $B$, et al. Mitral valve replacement with mechanical prosthesis in children: Improved operative risk and survival. Eur J Cardiothorac Surg. 2001;20:105-113.

10. Masuda $\mathrm{M}$, Kado $\mathrm{H}$, Matsumoto $\mathrm{T}$, Imoto $\mathrm{Y}$, Shiokawa $\mathrm{Y}$, Fukae $\mathrm{K}$, et al. Mitral valve replacement using bileaflet mechanical prosthetic valve in the first year of life. Jpn J Thorac Cardiovasc Surg. 2000;48:643-47.

11. Sim HT, Lee SC, Shin HJ, Park JJ, Yun TJ, Jhang WK, et al. Mitral valve replacement using mechanical prosthesis in children: Early and long term outcomes. Pediatr Cardiol, 2012;33: 639-645.

12. Alsoufi B, Manlhiot C, McCrindle B, Canver C, Sallehuddin $A$, Al-Oufi $S$, et al. Aortic and mitral valve replacement in children: is there any role for biologic and bioprosthetic substitutes? Eur J Cardiothorac Surg. 2009;36:84-90.

13. Alsoufi B, Manlhiot C, Al-Ahmadi M, McCrindle B, KaIloghlian A, Siblini G, et al. Outcomes and associated risk factors for mitral valve replacement in children. Eur J Cardiothorac Surg. 2011;40:543-51.

14. Alsoufi B, Manlhiot C, McCrindle B, Al-Halees Z, Sallehuddin A, Al-Oufi S, et al. Results after mitral valve replacement with mechanical prosthesis in young children. J Thorac Cardiovasc Surg. 2010;139:1189-96.

15. Calderone $C$, Raghuveer $G$, Hills $C h$, Atkins D, Burns T, Behrendt $D$, et al. Long-Term survival after mitral valve replacement in children aged < 5 years: A multi-institutional study. Circulation. 2001;104[suppl I]:I-143-I-47.

16. Wada N, Takahashi Y, Ando M, Park I, Kikuchi T. Mitral valve replacement in children under 3 years of age. Jpn J Thorac Cardiovasc Surg. 2005;53:545-50.

17. Tierney ES, Pigula FA, BerulChl, Lock JE, del Nido PJ, McElhinney DB. Mitral valve replacement in infants and children 5 years of age or younger: Evolution in practice and outcomes over three decades with a focus on supraannular prosthesis implantation. J Thorac Cardiovasc Surg. 2008;136:954-61. 